SU-ITP-93-37

$\mathrm{UG}-8 / 93$

hep-th/9401025

\title{
EXACT DUALITY
}

IN STRING EFFECTIVE ACTION

\author{
Eric Bergshoeff a and Ingeborg Entrop ? \\ Institute for Theoretical Physics, University of Groningen \\ Nijenborgh 4, 9747 AG Groningen, The Netherlands
}

Renata Kallosh

Physics Department, Stanford University, Stanford CA 94305

\begin{abstract}
We formulate sigma-model duality transformations in terms of spin connection. This allows to investigate the symmetry of the string action including higher order $\alpha^{\prime}$ corrections. An important feature of the new duality transformations is a simple homogeneous transformation rule of the spin connection (with torsion) and specifically adjusted transformation of the Yang-Mills field.

We have found that under certain conditions this duality is a symmetry of the full effective string action in the target space, free of $\alpha^{\prime}$ corrections. We demonstrate how the exact duality generates new fundamental string solutions from supersymmetric string waves.
\end{abstract}

\footnotetext{
${ }^{1}$ E-mail: bergshoe@th.rug.nl

${ }^{2}$ E-mail: entrop@th.rug.nl

${ }^{3}$ E-mail: kallosh@physics.stanford.edu
} 


\section{Introduction}

In recent years an active field of research has been the study of modified Einstein-Maxwell equations. The modifications that have been considered include additional scalar or antisymmetric tensor fields (called dilatons and axions) or modifications in which the electromagnetic field is replaced by non-Abelian Yang-Mills fields. These modified Einstein-Maxwell theories admit new solutions whose consistency crucially depends on the presence of the new fields and/or on the non-Abelian nature of the Yang-Mills fields. For examples of such new solutions see the review articles [1, 2, 3] and references therein.

One motivation for studying the above-mentioned modifications to Einstein-Maxwell theory is that they arise in string theory. The zero slope limit $\alpha^{\prime} \rightarrow 0$ of string theory corresponds to a modified Einstein-Maxwell theory of the type discussed above. The complete effective action also includes contributions which are of higher order in the Riemann tensor and the Yang-Mills field strength. Since string theory claims to give a consistent description of quantum gravity, solutions of the string effective action will contribute to our understanding of quantum gravity.

Not many exact solutions to the string equations of motion are known. One of the reasons for this is that knowledge about the explicit form of the higher order $\alpha^{\prime}$ corrections to the string effective action have become available only fairly recently [1]. The higher order terms in $\alpha^{\prime}$ in the effective action are for instance crucial in the construction of the five-brane soliton [5].

In general, it appears difficult to find exact solutions to the string equations of motion. Fortunately, if one considers spacetimes with a symmetry, there exist transformations which generate new solutions from old ones. Examples of solution generating transformations specific to the string effective action are the target space duality transformations [6, 7] and the symmetry transformations of [8].

So far, the target space duality transformations have only been derived in a sigma model formulation of string theory [6]. The explicit form of the duality transformation is only known to lowest order in $\alpha^{\prime}$. In general one expects that the duality transformations will be modified with an infinite number of terms of increasingly higher order in $\alpha^{\prime}$ but no information is available about these higher order corrections. Recently, Klimčík and Tseytlin found an exact duality between some pp-waves and flat space with non-vanishing antisymmetric tensor and dilaton fields [9]. A discussion, from the sigma model point of view, of other examples of situations where the duality transformations are exact can be found in [10, [11].

A discussion of less explicit examples of situations where the leading-order duality transfor-

mations do not acquire $\alpha^{\prime}$ corrections for a special choice of field redefinitions can also be found in [9].

The purpose of this paper is to find duality transformations which form a symmetry of the 
theory in the zero slope limit, and remain a symmetry of the theory even with account taken of $\alpha^{\prime}$ corrections. However, $\alpha^{\prime}$ corrections include terms involving spin connection. This forced us to formulate duality transformations not in terms of metric, as is usually done, but in terms of spin connection with torsion. The resulting transformations have a rather simple structure, which allowed us to investigate higher order $\alpha^{\prime}$ corrections.

We will also investigate special field configurations for which these duality transformations do not receive higher order corrections, i.e. are exact transformations to all orders in $\alpha^{\prime}$. To be specific, our starting point will be the supersymmetric string wave (SSW) solutions to tendimensional string theory [12]. These solutions are characterized by an arbitrary vector field $A_{\mu}$. It has been shown in [13 that, for the special case of plane fronted waves where only $A_{u} \neq 0$, these wave solutions are dual to the field outside a straight fundamental string [14]. Furthermore, it is known that the plane fronted waves are an exact solution of the string equations of motion [15, 16, 17]. We will show that the fundamental string (FS) solution is also an exact solution. As a consequence, we find that the duality transformation, applied to a plane fronted wave, is an exact transformation.

The above result can be generalized in the following way. We will derive a theorem stating that, given a field configuration that solves the zero slope limit of the string equations, the same field configuration can be promoted to an exact solution provided that all $\alpha^{\prime}$ corrections to the space-time supersymmetry transformation rules vanish. The latter condition is equivalent to the requirement that for our solution (i) the (Lorentz + Yang-Mills) Chern-Simons forms vanish and (ii) the so-called $T$-tensors (see eqs. (31)-(33)) vanish. In order to fulfill these two conditions, it will be necessary in certain cases to make a nontrivial Ansatz for the Yang-Mills gauge fieldst.

A corollary of our theorem is that, given two solutions to the zero slope limit of the string equations, which are connected to each other by a duality transformations, then the duality transformation is exact to all orders in $\alpha^{\prime}$ provided both solutions satisfy the two conditions in the theorem given above. The main application of our theorem in this paper will be to show that the SSW solutions, after duality, lead to a generalized FS solution, which is again an exact solution. Therefore, the duality transformation connecting the two solutions is exact to all orders in $\alpha^{\prime}$.

Our approach is different from the one developed in [8] in the treatment of the duality transformation of vector fields. We do not include vector fields in the zero slope limit of the effective action but treat the non-abelian vector fields at the level of $\alpha^{\prime}$ corrections. These corrections cancel against gravitational $\alpha^{\prime}$ corrections for the special configurations which we are considering.

The organization of this paper is as follows. In section 2 we will review the sigma model derivation of the target space duality transformations in the zero slope limit. As a new result we will also present the duality transformation of the Yang-Mills gauge fields since we will need

\footnotetext{
${ }^{4}$ Note that the Yang-Mills vector fields do not occur in the zero slope limit of the string effective action.
} 
them in the following. In section 3 we will explicitly show how the target space duality invariance works in the zero slope limit. In the next section this duality transformation will be applied to construct the generalized FS solution. In section 5 we will give the derivation of our theorem. In section 6 we will show that the FS solution is an exact solution and hence that the duality rotation connecting the SSW and generalized FS solution is exact. Details about our notations and conventions can be found in the Appendix A. Finally, in Appendices B and C we prove that the generalized FS solution and the solution of [9] are space-time supersymmetric.

\section{Sigma model duality}

We consider the sigma model action of the form

$$
S=\frac{1}{2 \pi} \int d^{2} z\left[\left(g_{\mu \nu}+B_{\mu \nu}\right) \partial x^{\mu} \bar{\partial} x^{\nu}+i \psi_{I}\left(\bar{\partial} \psi^{I}+V_{\mu}^{I K} \bar{\partial} x^{\mu} \psi_{K}\right)\right] .
$$

This action is a truncation of a supersymmetric sigma model [18 related to the heterotic string. We assume that the background fields $g_{\mu \nu}, B_{\mu \nu}$ and $V_{\mu}^{I K}$ are independent of some coordinate $x$ and may depend on all remaining bosonic coordinates $x^{\alpha}$, where $\left\{x^{\mu}\right\}=\left\{x, x^{\alpha}\right\}$. This sigma model allows a straightforward generalization of the discrete target space duality transformations rules [6] in presence of the vector fields in the background. We proceed in the standard way [19] by presenting the first order action

$$
\begin{aligned}
S_{1} & =\frac{1}{2 \pi} \int d^{2} z\left[g_{x x} A \bar{A}+\left(g_{x \alpha}+B_{x \alpha}\right) A \bar{\partial} x^{\alpha}+\left(\left(g_{\alpha x}+B_{\alpha x}\right) \partial x^{\alpha}+V_{x}\right) \bar{A}\right. \\
& \left.+\left(g_{\alpha \beta}+B_{\alpha \beta}\right) \partial x^{\alpha} \bar{\partial} x^{\beta}+i \psi_{I} \bar{\partial} \psi^{I}+V_{\alpha} \bar{\partial} x^{\alpha}+\tilde{\theta}(\partial \bar{A}-\bar{\partial} A)\right] .
\end{aligned}
$$

The following simplifying notation have been used

$$
\begin{aligned}
V_{x} & \equiv i \psi_{I} V_{x}^{I J} \psi_{J}, \\
V_{\alpha} & \equiv i \psi_{I} V_{\alpha}^{I J} \psi_{J} .
\end{aligned}
$$

By integrating out the Lagrange multiplier field $\tilde{\theta}$ on a topologically trivial world-sheet, one recovers the original action since the solution to the equation $(\partial \bar{A}-\bar{\partial} A)=0$ is $A=\partial x, \bar{A}=\bar{\partial} x$. It is important to stress that this dualization procedure requires the non-vanishing value of $g_{x x}$. If one integrates out the gauge fields $A, \bar{A}$ one gets the dual model

$$
\tilde{S}=\frac{1}{2 \pi} \int d^{2} z\left[\left(\tilde{g}_{\mu \nu}+\tilde{B}_{\mu \nu}\right) \partial \tilde{x}^{\mu} \bar{\partial} \tilde{x}^{\nu}+i \psi_{I}\left(\bar{\partial} \psi^{I}+\tilde{V}_{\mu}^{I K} \bar{\partial} \tilde{x}^{\mu} \psi_{K}\right)\right] .
$$

The new coordinates are $\left\{\tilde{x}^{\mu}\right\}=\left\{\tilde{\theta}, x^{\alpha}\right\}$ and the dual metric and antisymmetric tensor field are

$$
\begin{aligned}
\tilde{g}_{x x} & =1 / g_{x x}, \quad \tilde{g}_{x \alpha}=B_{x \alpha} / g_{x x} \\
\tilde{g}_{\alpha \beta} & =g_{\alpha \beta}-\left(g_{x \alpha} g_{x \beta}-B_{x \alpha} B_{x \beta}\right) / g_{x x} \\
\tilde{B}_{x \alpha} & =g_{x \alpha} / g_{x x}, \quad \tilde{B}_{\alpha \beta}=B_{\alpha \beta}+2 g_{x[\alpha} B_{\beta] x} / g_{x x} .
\end{aligned}
$$


The dual vector field is

$$
\tilde{V}_{x}^{J K}=-V_{x}^{J K} / g_{x x}, \quad \tilde{V}_{\alpha}^{J K}=V_{\alpha}^{J K}-\left(g_{x \alpha}+B_{x \alpha}\right) V_{x}^{J K} / g_{x x} .
$$

Taking into account the one-loop jacobian from integrating out $A, \bar{A}$-fields one finds, as usual, the dilaton transformation rules [6]

$$
\tilde{\phi}=\phi-\frac{1}{2} \log g_{x x}
$$

Thus by using the truncated version of a supersymmetric sigma model it is easy to supplement the well known target space duality transformations of the metric and of the antisymmetric tensor field and of the dilaton by the accompanying transformations of the vector fields.

\section{Target-Space Duality in the Zero Slope Limit}

Our starting point is the bosonic part of the action of $N=1, d=10$ supergravity [20]:

$$
S\left(g_{\mu \nu}, B_{\mu \nu}, \phi\right)=\frac{1}{2} \int d^{10} x \sqrt{-g} e^{-2 \phi}\left(-R+4(\partial \phi)^{2}-\frac{3}{4} H^{2}\right)
$$

where $g_{\mu \nu}$ is the metric, $\phi$ the dilaton and $H$ the field strength of the axion $B_{\mu \nu}$. We now consider the special class of field configurations which have an isometry generated by a Killing vector $k^{\mu}$. It is convenient to use a special coordinate system where $k^{\mu}$ is constant and is only nonzero in one direction, let us say the $x$ direction. Furthermore we assume that $k^{\mu}$ is a non-null Killing vector, i.e. $k^{2} \neq 0$. The isometry property then amounts to the following condition on the field configuration $\left\{g_{\mu \nu}, B_{\mu \nu}, \phi\right\}$ :

$$
k^{\mu} \partial_{\mu}\left\{g_{\mu \nu}, B_{\mu \nu}, \phi\right\}=\partial_{x}\left\{g_{\mu \nu}, B_{\mu \nu}, \phi\right\}=0 .
$$

We want to show that the action (8) is invariant under target space duality transformations modulo terms which contain the derivative of one of the fields with respect to $x$. In other words we want to show that

$$
S\left(g_{\mu \nu}, B_{\mu \nu}, \phi\right)=S\left(\tilde{g}_{\mu \nu}, \tilde{B}_{\mu \nu}, \tilde{\phi}\right)+\int d^{10} x A(g, B, \phi) \partial_{x} B(g, B, \phi),
$$

where $A$ and $B$ are some expressions in terms of $g_{\mu \nu}, B_{\mu \nu}$ and $\phi$. If this is the case for any field configuration that satisfies (9) then the target-space duality transformation serves as a truly solution generating transformation: the dual of any solution of the field equations that is

\footnotetext{
${ }^{5}$ Strictly speaking, we should take in (7/7) the absolute value $\left|g_{x x}\right|$ instead of $g_{x x}$.

${ }^{6}$ We use the same conventions as in [12], except that we have redefined the axion field with $B_{\mu \nu} \rightarrow-\frac{3}{2} B_{\mu \nu}$ in order to agree with the duality transformations given in the previous section. Further details about our notation and conventions can be found in the appendix of [12].
} 
independent of the coordinate $x$ will automatically be another, inequivalent, solution of the same field equations.

In order to show that the action (8) is invariant under the duality transformations given in the previous section it is convenient to use the zehnbein instead of the metric. The zehnbein transforms under the following duality transformations:

$$
\begin{aligned}
\tilde{e}_{x}^{a} & =\frac{1}{g_{x x}} e_{x}^{a} \\
\tilde{e}_{\alpha}^{a} & =e_{\alpha}^{a}-\frac{1}{g_{x x}}\left(g_{x \alpha}-B_{x \alpha}\right) e_{x}^{a} .
\end{aligned}
$$

From the point of view of reproducing the duality transformation of the metric, given in equation (5), we could equally well use a different duality transformation for the zehnbeins. We could have used the transformation

$$
\tilde{e}_{x}^{a}=-\frac{1}{g_{x x}} e_{x}^{a}, \quad \tilde{e}_{\alpha}^{a}=e_{\alpha}^{a}-\frac{1}{g_{x x}}\left(g_{x \alpha}+B_{x \alpha}\right) e_{x}^{a} .
$$

This transformation coincides exactly with the duality transformation of the vector field in equation (6). We have found, however, that to provide the absence of $\alpha^{\prime}$ corrections we have to use the duality transformation of the zehnbein given in equation (11) if the one for the vector field is given in equation (6). Another possibility is to change both of them to the opposite one. This will be a necessary condition for the duality symmetry to preserve the embedding condition of the spin connection into a subgroup of the gauge group.

Under a duality transformation the spin-connection and the axion field strength transform as:

$$
\begin{aligned}
\tilde{\omega}_{c}{ }^{a b} & =\omega_{c}{ }^{a b}-\frac{1}{k^{2}} k_{c} k^{d} \Omega_{d-}{ }^{a b}-\frac{2}{k^{2}} k^{d} k^{[a} \Omega_{d-, c}{ }^{b]} \\
\tilde{H}_{a b c} & =H_{a b c}-\frac{2}{k^{2}} k^{d} k_{[a} \Omega_{d-, b c]}
\end{aligned}
$$

where the torsionful spin connections $\Omega_{\mu \pm}^{a b}$ are defined by

$$
\Omega_{\mu \pm}^{a b}=\omega_{\mu}^{a b}(e) \mp \frac{3}{2} H_{\mu}^{a b} .
$$

For later convenience we note that the dual of the torsionful spin connections are given by

$$
\begin{aligned}
& \tilde{\Omega}_{c-}{ }^{a b}=\Omega_{c-}{ }^{a b}-\frac{2}{k^{2}} k_{c} k^{d} \Omega_{d-}{ }^{a b}, \\
& \tilde{\Omega}_{c+}{ }^{a b}=\Omega_{c+}{ }^{a b}-\frac{4}{k^{2}} k^{d} k^{[a} \Omega_{d-, c}{ }^{b]} .
\end{aligned}
$$

\footnotetext{
${ }^{7}$ Note that with this definition the $\Omega_{ \pm}$of this paper is identical to the $\Omega_{ \pm}$of 12 after the redefinition $B_{\mu \nu} \rightarrow$ $-\frac{3}{2} B_{\mu \nu}$
} 
Using the identity

$$
-\int d^{10} x \sqrt{-g} e^{-2 \phi} R=\int d^{10} x \sqrt{-g} e^{-2 \phi}\left(\omega_{a}^{a c} \omega_{b}^{b c}+\omega_{a}^{b c} \omega_{b}^{c a}+4\left(\partial_{a} \phi\right) \omega_{b}^{b a}\right),
$$

we can now rewrite the action (\$) in the following convenient form:

$$
S=\frac{1}{2} \int d^{10} x e e^{-2 \phi}\left(4\left(\partial^{a} \phi+\frac{1}{2} \omega_{b}^{b a}\right)^{2}+\omega_{a}^{b c} \omega_{b}^{c a}-\frac{3}{4} H_{a b c} H^{a b c}\right)
$$

where $e=\operatorname{det} e_{\mu}^{a}$. Using the additional identity

$$
k^{b} k^{c} \omega_{b, c a}=-\frac{1}{2} \partial_{a} k^{2}
$$

and transformation rule

$$
\tilde{e}=\frac{1}{k^{2}} e,
$$

it is straightforward to show that the action $(\mathbb{1 7})$ is invariant under target-space duality transformations. We note that the action consists of three parts which are separately duality invariant: the combinations $e e^{-2 \phi},\left(\partial^{a} \phi+\frac{1}{2} \omega_{b}^{b a}\right)$ and $\left(\omega_{a}^{b c} \omega_{b}^{c a}-\frac{3}{4} H_{a b c} H^{a b c}\right)$ are all three duality invariant.

Thus we have shown that the target space action in the zero slope limit is invariant under the sigma model duality transformations, given in equations (5) and (7). The vector fields are not present in the effective action in the zero slope limit.

\section{New Solutions in the Zero Slope Limit}

The purpose of this section is to show how the duality transformations can be used to generate new solutions in the zero slope limit. Higher-order $\alpha^{\prime}$ corrections will be considered in the next two sections. Our starting point is the Supersymmetric String Wave (SSW) solution of [12]. In 12 it was shown that the SSW solves the string equations of motion to all orders in $\alpha^{\prime}$. Here we will only consider the zero slope limit. In particular, we will set the vector gauge fields equal to zero. The solution thereby reduces to the one given in [21], [22]. A crucial feature of the SSW solution is the existence of a null Killing vector $l^{\mu}$ with $l^{2}=0$. This Killing vector generates an isometry in the $v$ direction where we use light-cone coordinates $x^{\mu}=\left(u, v, x^{i}\right)(i=1, \ldots, 8)$. Since this Killing vector is null we cannot use it for a duality transformation. We therefore make the additional assumption that the fields occurring in the SSW solution are also independent of the $u$ coordinate. Since the Ansatz for the dilaton in the SSW solution only depends on $u$, it must be a constant and for simplicity we will take this constant to be zero. The remaining nonzero fields $g_{\mu \nu}$ and $B_{\mu \nu}$ are both described in terms of one vector function of the transverse coordinates $x^{i}$ :

$$
A_{\mu}\left(x^{i}\right)=\left\{A_{u}\left(x^{i}\right), A_{v}=0, A_{i}\left(x^{i}\right)\right\}
$$


They are given by the Brinkmann metric [23] and the following 2-form》:

$$
\begin{aligned}
d s^{2} & =2 d u d v+2 A_{\mu} d x^{\mu} d u-d x^{i} d x^{i} \\
B & =2 A_{\mu} d x^{\mu} \wedge d u
\end{aligned}
$$

The equations that $A_{u}\left(x^{i}\right)$ and $A_{i}\left(x^{i}\right)$ have to satisfy are:

$$
\triangle A_{u}=0, \quad \triangle \partial^{[i} A^{j]}=0
$$

where the Laplacian is taken over the transverse directions only. Since $g_{\mu \nu}$ and $B_{\mu \nu}$ are independent of $u$ and $v$ they are independent of $x$ and $t$. For our duality transformation we will use the isometry in the space-like $x$ direction. The non-null Killing vector generating this isometry will be denoted by $k^{\mu}$. Note that we have now two isometries given by

$$
l^{\mu} \partial_{\mu}\left\{g_{\mu \nu}, B_{\mu \nu}\right\}=\partial_{v}\left\{g_{\mu \nu}, B_{\mu \nu}\right\}=0, \quad k^{\mu} \partial_{\mu}\left\{g_{\mu \nu}, B_{\mu \nu}\right\}=\partial_{x}\left\{g_{\mu \nu}, B_{\mu \nu}\right\}=0 .
$$

A straightforward application of the sigma-model duality transformations given in (5), (7)) on the SSW solution given in eq. (21) leads to the following new solution of the zero slope limit equations of motion:

$$
\begin{aligned}
d s^{2} & =-\left(A_{u}-1\right)^{-1}\left\{2 d u d v+2 A_{i} d u d x^{i}\right\}-d x^{i} d x^{i} \\
B & =\left(A_{u}-1\right)^{-1}\left\{2 A_{u} d u \wedge d v+2 A_{i} d u \wedge d x^{i}\right\} \\
\phi & =-\frac{1}{2} \log \left(A_{u}-1\right)
\end{aligned}
$$

Note that we can make the following particular choice of the vector function $A_{\mu}$ ?:

$$
A_{u}=-\frac{\tilde{c} M}{r^{6}}, \quad A_{i}=0
$$

where $r^{2}=x^{i} x_{i}$ and $\tilde{c}$ a constant. The solution given in (24) reduces then to the solution of [14] corresponding to the field outside a fundamental string with total mass $M$. We will hence refer to the solution (24) as the generalized FS solution 1 .

It was shown in [12 that the SSW solution is supersymmetric under 8 of the 16 ten-dimensional supersymmetries. In 114, it has been shown that the dual FS solution, for the special case that $A_{i}=0$, is again supersymmetric. In Appendix B we will show that also the generalized FS solution, with $A_{i} \neq 0$, is supersymmetric.

\footnotetext{
${ }^{8}$ We use the following form notation: $d s^{2}=g_{\mu \nu} d x^{\mu} d x^{\nu}$ and $B=B_{\mu \nu} d x^{\mu} \wedge d x^{\nu}$.

${ }^{9}$ In order to solve the equations (22), it is understood that a source term at $r=0$, representing a fundamental string, has been added to these equations.

${ }^{10}$ Note that the solution (24) does not yet include the vector fields. The complete generalized FS solution, including the vector fields, is given in (54).
} 


\section{$5 \quad \alpha^{\prime}$ corrections}

In this section we will consider the $\alpha^{\prime}$ corrections to the zero slope limit. In particular, we will derive a theorem stating that any solution to the zero slope limit string equations can be promoted to an exact solution to all orders in $\alpha^{\prime}$ provided that (i) the $T$-tensors defined by eqs. (31) - (33) vanish and (ii) a particular combination of the (Lorentz + Yang-Mills) ChernSimons terms vanish. We note that the discussion in this section is independent of any particular solution to the field equations.

There exists one remarkable property of the duality transformations considered above, which is of crucial importance for the understanding of $\alpha^{\prime}$ corrections. This property is best see in terms of the duality transformations of the torsionful spin connections $\Omega_{c-}{ }^{a b}$, defined above in eq. (14). The useful form of this transformation is

$$
\tilde{\Omega}_{c-}{ }^{a b}=\Pi_{c}{ }^{d} \Omega_{d-}{ }^{a b},
$$

where we have introduced the projector

$$
\Pi_{c}^{d} \equiv \delta_{c}{ }^{d}-\frac{2}{k^{2}} k_{c} k^{d}
$$

The square of this projector is a unit operator:

$$
\Pi_{c}^{d} \Pi_{d}^{e}=\delta_{c}^{e} .
$$

This property of the projector nicely confirms the fact that we are performing a discrete operation and that two such operations bring us back to the original configuration since

$$
\tilde{\widetilde{\Omega}}_{c-}{ }^{a b}=\Pi_{c}{ }^{d} \tilde{\Omega}_{d-}{ }^{a b}=\Pi_{c}{ }^{d} \Pi_{d}{ }^{e} \Omega_{e-}{ }^{a b}=\Omega_{c-}{ }^{a b} .
$$

Now let us consider the $\alpha^{\prime}$ corrections to the string effective action. It is well known that one has to add to $S^{(0)}$ the Lorentz and Yang-Mills Chern-Simons terms which play a crucial role in the Green-Schwarz anomaly cancellation mechanism $\amalg$. These terms break supersymmetry. To restore supersymmetry order by order in $\alpha^{\prime}$, one has to add to $S^{(0)}$ an infinite series of higher order terms in $\alpha^{\prime}$. By the procedure of adding terms to restore supersymmetry, the effective action was obtained in [12] up to order $O\left(\alpha^{\prime 4}\right)$ terms:

$$
\begin{aligned}
S= & \frac{1}{2} \int d^{10} x \sqrt{-g} e^{-2 \phi}\left(-R+4(\partial \phi)^{2}-\frac{3}{4} H^{2}+\right. \\
& \left.+\frac{1}{2} T+2 \alpha^{\prime} T^{\mu \nu} T_{\mu \nu}+6 \alpha^{\prime} T^{\mu \nu \lambda \rho} T_{\mu \nu \lambda \rho}+O\left(\alpha^{\prime 4}\right)\right),
\end{aligned}
$$

\footnotetext{
11 Not much is known about the properties of $\alpha^{\prime}$ corrections, which are not related to anomalies. For some recent results about these additional corrections, see [24] and references therein. We will not consider these corrections in this paper.
} 
where the antisymmetric tensor $T_{\mu \nu \lambda \rho}$, the symmetric tensor $T_{\mu \nu}$, and the scalar $T$ are given by

$$
\begin{aligned}
T_{\mu \nu \lambda \rho} & =2 \alpha^{\prime}\left(R_{[\mu \nu}^{a b}\left(\Omega_{-}\right) R_{\lambda \rho]}^{a b}\left(\Omega_{-}\right)+\frac{1}{30} \operatorname{tr} F_{[\mu \nu} F_{\lambda \rho]}\right), \\
T_{\mu \nu} & =2 \alpha^{\prime}\left(R_{\mu \lambda}^{a b}\left(\Omega_{-}\right) R_{\nu}^{\lambda}{ }^{a b}\left(\Omega_{-}\right)+\frac{1}{30} \operatorname{tr} F_{\mu \lambda} F_{\nu}^{\lambda}\right), \\
T & =T_{\mu}^{\mu} .
\end{aligned}
$$

In the above expression there are explicit and implicit $\alpha^{\prime}$ corrections. The explicit corrections always appear via $T$-tensors, and they are essentially the $\alpha^{\prime}$ factors in front of eqs. (31)-(33). The implicit $\alpha^{\prime}$ corrections always appear via the torsion $H$ which is defined by the following iterative procedure: At the lowest order $H$ is just $H_{[\mu \nu \rho]}^{(0)}=\partial_{[\mu} B_{\nu \rho]}$. With $H^{(0)}$ we calculate the lowest order $\Omega_{ \pm}=\Omega_{ \pm}^{(0)}$, as given in eq. (14). At first order in $\alpha^{\prime}, H=H^{(1)}$ is $H^{(0)}$ corrected by the Yang-Mills Chern-Simons term and the Lorentz Chern-Simons term corresponding to the zero-order $\Omega_{-}=\Omega_{-}^{(0)}$ :

$$
H_{\mu \nu \rho}^{(1)}=\partial_{[\mu} B_{\nu \rho]}+\alpha^{\prime}\left(\omega_{\mu \nu \rho}^{Y . M .}+\omega_{\mu \nu \rho}^{L}\right)
$$

where

$$
\omega_{\mu \nu \rho}^{Y . M .}=-\frac{1}{5} \operatorname{tr}\left\{V_{[\mu} \partial_{\nu} V_{\rho]}-\frac{1}{3} V_{[\mu} V_{\nu} V_{\rho]}\right\}
$$

and

$$
\omega_{\mu \nu \rho}^{L}=-6\left\{\Omega_{[\mu-}^{(0) a b} \partial_{\nu} \Omega_{\rho]-}^{(0) a b}-\frac{1}{3} \Omega_{[\mu-}^{(0) a b} \Omega_{\nu-}^{(0) a c} \Omega_{\rho-]}^{(0) c b}\right\} .
$$

With $H^{(1)}$ one would get $\Omega^{(1)}$ using again its definition eq. (14) and $H^{(2)}$ would be given by the above expression but with $\Omega^{(0)}$ replaced by $\Omega^{(1)}$. Iterating this procedure one gets the all-order expression $H$ for the torsion which involves the promised infinite series of corrections.

In short, to be able to understand the properties of $\alpha^{\prime}$ corrections to specific configurations we have to calculate the value of the Lorentz and Yang-Mills Chern-Simons term and the values of all $T$-tensors, presented above.

To study the corrections to the equations of motion we will use the corresponding analysis, performed in [12]. We first have to vary the action (30) over all the fields present in the theory, and only then substitute the solutions in the corrected equations. We will study the linear corrections separately. The equations of motion corrected up to first order come from the action

$$
S^{(1)}=\frac{1}{2} \int d^{10} x \sqrt{-g} e^{-2 \phi}\left(-R+4(\partial \phi)^{2}-\frac{3}{4} H^{2}+\frac{1}{2} T\right) .
$$

All terms of order $\alpha^{\prime 2}$ and higher are neglected at this stage. The corrections linear in $\alpha^{\prime}$ to the lowest order equations of motion are derived from the variation $\delta\left(S^{(1)}-S^{(0)}\right) \equiv \delta \Delta S$. It is convenient to perform this variation with respect to the explicit dependence of the action on 
$g_{\mu \nu}, V_{\mu}, \phi$ and $B_{\mu \nu}$, and then with respect to the implicit dependence on these fields through the torsionful spin connection $\Omega_{-}$, that is

$$
\begin{aligned}
\delta \Delta S= & \frac{\delta \Delta S}{\delta g_{\mu \nu}} \delta g_{\mu \nu}+\frac{\delta \Delta S}{\delta B_{\mu \nu}} \delta B_{\mu \nu}+\frac{\delta \Delta S}{\delta \phi} \delta \phi+ \\
& +\frac{\delta \Delta S}{\delta V_{\mu}} \delta V_{\mu}+\frac{\delta \Delta S}{\delta \Omega_{\mu-}{ }^{a b}} \delta \Omega_{\mu-}{ }^{a b}
\end{aligned}
$$

where

$$
\delta \Omega_{\mu-}{ }^{a b}=\frac{\delta \Omega_{\mu-}{ }^{a b}}{\delta g_{\mu \nu}} \delta g_{\mu \nu}+\frac{\delta \Omega_{\mu-}{ }^{a b}}{\delta B_{\mu \nu}} \delta B_{\mu \nu}+\frac{\delta \Omega_{\mu-}{ }^{a b}}{\delta V_{\mu}} \delta V_{\mu} .
$$

The explicit variations are

$$
\begin{aligned}
\frac{\delta \Delta S}{\delta g_{\mu \nu}}= & -\frac{1}{4} \sqrt{-g} e^{-2 \phi}\left(T^{\mu \nu}-g^{\mu \nu} T\right) \\
\frac{\delta \Delta S}{\delta \phi}= & -\frac{1}{2} \sqrt{-g} e^{-2 \phi} T \\
\frac{\delta \Delta S}{\delta B_{\mu \nu}}= & \frac{3}{4} \partial_{\lambda}\left[\sqrt{-g} e^{-2 \phi}\left(H^{(1) \lambda \mu \nu}-H^{(0) \lambda \mu \nu}\right)\right] \\
\frac{\delta \Delta S}{\delta V_{\mu}}= & \frac{1}{15} \alpha^{\prime}\left\{\partial_{\lambda}\left(\sqrt{-g} e^{-2 \phi} F^{\lambda \mu}\right)+\sqrt{-g} e^{-2 \phi}\left[V_{\lambda}, F^{\lambda \mu}\right]+\right. \\
& \left.-\frac{3}{2} \sqrt{-g} e^{-2 \phi} H_{\lambda \rho}^{(0) \mu} F^{\lambda \rho}+\frac{3}{2} V_{\rho} \partial_{\lambda}\left(\sqrt{-g} e^{-2 \phi} H^{(0) \lambda \mu \rho}\right)\right\} .
\end{aligned}
$$

If some solution of the zero slope limit equations has the property that the $T$-tensors vanish on this solution, the first two equations above do not get linear $\alpha^{\prime}$ corrections. The third equation is not corrected, provided the combination of Lorentz and Yang-Mills Chern-Simons term is vanishing for the configuration. The last equation is satisfied if the classical equation of motion for the vector field [2] as well as the equation of motion for the $B_{\mu \nu}$-field in the zero slope limit are satisfied. We next consider the implicit variations represented by the last term in eq. (38). In [4] a rather non-trivial property of the string effective action was analysed. This property is that all the implicit variations are proportional to the lowest order equations of motion of the different fields [3]. We therefore conclude that, to linear order in $\alpha^{\prime}$, any solution of the lowest order equations of motion are also solutions of the equations of motion corrected to order $\alpha^{\prime}$ provided that (i) the T-tensor $T_{\mu \nu}$ and (ii) the (Lorentz + Yang-Mills) Chern-Simons terms vanish.

Now let us consider the higher order $\alpha^{\prime}$ corrections. The general structure of the bosonic part of the effective action, which can be obtained by the procedure outlined before based upon the restoration of supersymmetry, has been conjectured in ref. [12]. New terms in the action

\footnotetext{
${ }^{12}$ One can include the vector field action in the classical Lagrangian of supergravity interacting with the YangMills multiplet. We treated the vector field here as coming at the level of $\alpha^{\prime}$ corrections, which is natural in the framework of string effective action.

${ }^{13}$ For more details, see $[$ ] and the Appendix of [12].
} 
are quadratic or of higher degree in the $T$-tensors. Therefore their contribution to the string equations of motion automatically vanishes for the configurations with vanishing $T$-tensors. This concludes the proof of the theorem stated at the beginning of this section.

Finally, we would like to note that the vanishing of a combination of Lorentz and Yang-Mills Chern-Simons term in $H_{\mu \nu \lambda}$ and the vanishing of all $T$-tensors is sufficient for the absence of corrections to the supersymmetry transformation laws and the Killing spinors [12], [4]. As a corollary of this we conclude that the condition for a field configuration that solves the zero slope limit equations of motion to be exact, i.e. to be free of $\alpha^{\prime}$-corrections, coincides with the property of the configuration to have vanishing $\alpha^{\prime}$-corrections to classical supersymmetry transformations.

\section{Exact duality between pp-waves and strings}

The complete discussion of the supersymmetric string waves and $\alpha^{\prime}$ corrections has been performed in [12]. Our final conclusion was that the on-shell action, the fields that solve the lowest order equations of motion and the Killing spinors for the SSW solutions do not receive any higher order string corrections.

In this section we would like to use the pp-wave solution, i.e. the SSW solution with $A_{i}=0$, as the starting point for investigation of the corrections to duality symmetry in the target space. In the next section we will consider the general case with $A_{i} \neq 0$.

Gravitational plane fronted waves [23] have a null Killing vector $\nabla_{\mu} l_{\nu}=0, \quad l^{\nu} l_{\nu}=0$ and very special dependence of this null vector. This simplifies the analysis of higher order corrections to field equations.

Consider the class of 10-dimensional pp-waves with metrics of the form

$$
d s^{2}=2 d u d v+K\left(u, x^{i}\right) d u^{2}-d x^{i} d x^{i}
$$

where $i=1,2, \ldots, 8$, the Riemann curvature is [17, 22]

$$
R_{\mu \nu \rho \sigma}=-2 l_{[\mu}\left(\partial_{\nu]} \partial_{[\rho} K\right) l_{\sigma]} .
$$

The curvature is orthogonal to $l_{\mu}$ in all its indices. This fact is of crucial importance in establishing that all higher order in $\alpha^{\prime}$ terms in the equations of motion are zero due to the vanishing of all the possible contractions of curvature tensors. The dilaton, the antisymmetric tensor field and

the vector field are absent in this solution. The only non-trivial function in the metric has to satisfy the equation

$$
\triangle K=0
$$


where $\triangle=\partial_{i} \partial_{i}$ is the flat space Laplacian. The spin connection of these pp-waves is given by the following expression:

$$
\omega_{\mu}{ }^{a b}=-l_{\mu} l^{[a} \partial^{b]} K .
$$

Note also that the indices $a b$ related to the fact that the spin connection is a Lorentz-Lie-algebra valued object

$$
\omega \equiv d x^{\mu} \omega_{\mu}^{a b} M_{a b}=-d x^{\mu} l_{\mu} l^{[a} \partial^{b]} K M_{a b}
$$

have at least one null vector. In this case the spin connection coincides with the torsionful spin connection since the antisymmetric field strength tensor $H_{a b c}$ vanishes:

$$
\Omega_{c-}{ }^{a b}=-l_{c} l^{[a} \partial^{b]} K .
$$

This spin connection is orthogonal to $l_{a}$ in all indices and what is very important, has at least one $l$-vector in $a b$-type indices.

In the discussion below we also have to take into account that

$$
\partial_{\mu} l^{a}=0 .
$$

The structures which we need, Lorentz Chern-Simons term given in eq. (36) and all T-tensors, given in eqs. (31)-(33), always have all $a b$-type indices contracted. This means that for the ppwaves all these structures vanish. There is no way to contract those null vectors, if they are contracted with another $l$ we get zero and if they are contracted with $\partial$ we have zero again, since this a Killing condition, the solution is independent of $v$. Thus for pp-waves the $\alpha^{\prime}$ corrections to the action, to the equation of motion and to supersymmetric transformations of all fermion fields vanish.

As explained in [13] the dual version of pp-waves (without a dilaton, antisymmetric field and vector field) is a string solution of [14]. As explained above, the pp-waves have no $\alpha^{\prime}$ corrections since the Lorentz Chern-Simons term and all T-tensors vanish for pp-waves. Now that we have a simple duality transformation rule for the torsionful spin connection we can investigate the corresponding features of the solution dual to the pp-waves.

As stated in the beginning of the previous section, the duality transformation $\tilde{\Omega}_{c-}{ }^{a b}=$ $\Pi_{c}{ }^{d} \Omega_{d-}{ }^{a b}$ does not affect the structure of the $a b$-type indices in this expression. Therefore the proof that the Lorentz Chern-Simons term and all T-tensors vanish for pp-waves is immediately extended to the dual version, i.e. to the string solution of [14]. The reason for that is that all those terms always have at least one null Killing vector in $a$ or $b$ direction, which has to be contracted with another $a$ - or $b$-type index. Although the contraction with new Killing vector $k^{\mu}$ is possible in principle and would give a non-vanishing contribution since $k^{2} \neq 0$ and $k \cdot l \neq 0$, we see from the structure of Chern-Simons term and T-tensors that after duality transformation no such contraction actually occurs.

Having established the vanishing of $\alpha^{\prime}$ corrections to Lorentz Chern-Simons term and all Ttensors for the FS string solution of [14] we may apply the general analysis performed in the 
previous section. We find that there are no corrections to the FS solution of the zero slope limit equations. We therefore conclude that both the FS solution of [14] and the pp-waves are exact solutions of the string effective action.

A simplifying feature of the pp-waves is the fact that they cannot have corrections independently of the specific form of the $\alpha^{\prime}$ corrections. For the dual version, the FS solution, we have under complete control only the $\alpha^{\prime}$ corrections described above which are related to anomalies. A priori they could receive an infinite set of corrections, in principle, unless the iterative procedure requires them to vanish on each step as we have seen above.

The conclusion of this section is that the duality transformation, which relates the pp-waves to the FS solution is an exact duality transformation, i.e. the $\alpha^{\prime}$ corrections to the duality transformation vanish for this case.

\section{$7 \quad$ Exact duality between supersymmetric string waves and generalized FS solutions}

In this section we extend the discussion of the previous section to the generalized FS solution given in eq. (24) for the case that $A_{i} \neq 0$. It has been shown in [12] that even for this case the corresponding SSW solution is an exact solution to all orders in $\alpha^{\prime}$. This is a nontrivial result since with $A_{i} \neq 0$ both the Lorentz Chern-Simons term as well as the $T$ tensors do not vanish. To make both vanish one must make a nontrivial Ansatz for the vector gauge fields $V_{\mu}{ }^{I J}$ and embed the torsionful spin connection $\Omega_{-}$in an $S O(8)$ subgroup of the gauge group, thereby identifying the spin connection with the gauge field:

$$
\frac{1}{\sqrt{30}} V_{\mu}^{I J}=l_{\mu} V^{I J} \equiv \Omega_{\mu-}^{a b}=l_{\mu} \mathcal{A}^{a b} \quad(a, b, I, J=1, \ldots, 8)
$$

Here the Yang-Mills indices refer to the adjoint representation of $S O(8)$. One then makes use of the fact that with a non-zero gauge field the Lorentz Chern-Simons term always occurs together with a Yang-Mills Chern-Simons term. The same applies to the $T$ tensors where the $R^{2}$ terms are always accompanied by similar $F^{2}$ terms. The above identification then leads to a cancellation between the spin connection and gauge field terms such that even with $A_{i} \neq 0$ the (Lorentz + Yang-Mills ) Chern-Simons terms and the (generalized) $T$ tensors do vanish. Of course this cancellation only involves terms with the function $A_{i}$. The terms involving $A_{u}$ in the Lorentz sector already cancel by themselves as has been shown in the previous section.

To investigate what happens with the (Lorentz + Yang-Mills) Chern-Simons terms and the $T$ tensors for the generalized FS solution we apply a duality transformation on the torsionful spin connection corresponding to the SSW solution. This leads to the following expression of $\Omega_{-}$for 
the generalized FS solution:

$$
\tilde{\Omega}_{c-}{ }^{a b}=\left\{l_{c}-\frac{2 k \cdot l}{k^{2}} k_{c}\right\} \mathcal{A}^{a b}=\Pi_{c}{ }^{d} l_{d} \mathcal{A}^{a b} .
$$

Note that the structure of the $a b$ indices remains unchanged under a duality rotation.

In order to show that the generalized FS solution is again a solution of the field equations to all orders in $\alpha^{\prime}$ it is essential that the above-mentioned cancellation between the spin connection and gauge field terms in the (Lorentz + Yang-Mills) Chern-Simons terms and the $T$ tensors is not spoiled by the duality transformation. We therefore require that the embedding is duality invariant. In order to obtain a duality-invariant embedding we want that $\Omega_{-}$transforms in the same way as the Yang-Mills gauge fields. Luckily enough it turns out that this is indeed the case. Using the duality transformation of the vector fields given in (6) one can show that the duality transformations of the spin connection and the gauge fields have precisely the same form: ${ }^{\text {Tt }}$ :

$$
\tilde{\Omega}_{c-}{ }^{a b}=\Pi_{c}{ }^{d} l_{d} \mathcal{A}^{a b} \quad \underline{\text { and }} \quad \frac{1}{\sqrt{30}} \tilde{V}_{c}{ }^{I J}=\Pi_{c}{ }^{d} l_{d} \mathcal{A}^{I J} .
$$

This means that the embedding condition (51) is indeed duality invariant and the cancellations which took place between the spin connection and gauge field terms in the SSW solution again take place, after the duality rotation, for the generalized FS solution. Hence, for the generalized FS solution we can again derive that the (Lorentz + Yang-Mills) Chern-Simons terms and the $T$-tensors are zero. At this point we can use the results of section 5 where we have shown that the vanishing of the (Lorentz + Yang-Mills) Chern-Simons form and the $T$ tensors is enough to ensure that the zero-slope limit solution extends to a solution to all orders in $\alpha^{\prime}$.

The conclusion is therefore that the generalized FS solution given by

$$
\begin{aligned}
d s^{2} & =-\left(A_{u}-1\right)^{-1}\left\{2 d u d v+2 A_{i} d u d x^{i}\right\}-d x^{i} d x^{i}, \\
B & =\left(A_{u}-1\right)^{-1}\left\{2 A_{u} d u \wedge d v+2 A_{i} d u \wedge d x^{i}\right\}, \\
\phi & =-\frac{1}{2} \log \left(A_{u}-1\right), \\
V_{\mu}^{I J} & =-\left(A_{u}-1\right)^{-1} l_{\mu} \mathcal{A}^{I J} \quad(I, J=1, \ldots, 8),
\end{aligned}
$$

with $\mathcal{A}_{\mu \nu}=\partial_{\mu} A_{\nu}-\partial_{\nu} A_{\mu}$ solves the string equations of motion to all orders in $\alpha^{\prime}$. The equations that $A_{u}\left(x^{i}\right)$ and $A_{i}\left(x^{i}\right)$ have to satisfy are:

$$
\triangle A_{u}=0, \quad \triangle \partial^{[i} A^{j]}=0,
$$

where the Laplacian is taken over the transverse directions. Furthermore, the duality transformation connecting the SSW solution and the generalized FS solution is exact to all orders in $\alpha^{\prime}$.

\footnotetext{
${ }^{14}$ In the equations given below it is understood that the SSW metric is used. Note that the dual gauge fields are given with flat indices. To convert them into curved indices one should use the metric corresponding to the FS solution.
} 


\section{Conclusion}

In this paper we presented a set of duality transformations for the zehnbein, spin connection (with torsion) and vector fields. They are given in equations (5), (6), (11), (15). The most elegant duality transformation of the spin connection with torsion is given in equation (26). This specific transformation plays a crucial role in the analysis of $\alpha^{\prime}$ corrections.

We have found the conditions under which a target space duality symmetry is exact, i.e. does not acquire $\alpha^{\prime}$ corrections. Two configurations can be qualified as being exactly dual to each other if:

i) there exists a non-null Killing vector in the original as well as in the dual configuration, which allows one to identify the corresponding sigma-model duality transformation. This transformation defines a symmetry of the zero slope limit of the string effective action in the target space.

ii) the condition for the action and equations of motion not to acquire $\alpha^{\prime}$ corrections is provided by the vanishing of the combination of the Lorentz and Yang-Mills Chern-Simons term as well as by the vanishing of the $T$-tensors for the original as well as for the dual configuration.

iii) exact duality in all explicit examples known to us brings one supersymmetric configuration to another supersymmetric configuration. The zero slope supersymmetric transformation rules are not affected by the $\alpha^{\prime}$ corrections for the original as well as the final configurations related by exact duality.

Exact duality defined above serves as an exact solution generating transformation. As an example of configurations related by exact duality we have analysed the pp-waves and fundamental string solutions [14]. Both solutions are free of $\alpha^{\prime}$ corrections 10.

As a further application of our general results we have also checked that the examples of exact duality investigated by Klimčík and Tseytlin [9] also have some unbroken space-time supersymmetry. Their original configuration is supersymmetric, since it is equivalent to one of the Güven 15] solutions. The dual configuration turns out to be also supersymmetric, as we have shown in Appendix C.

A more general example of exact duality is given by the supersymmetric string waves [12] and the generalized fundamental strings given in (54). The last solution to the best of our knowledge is new. The proof that it is free of anomaly related $\alpha^{\prime}$ corrections is provided by the corresponding properties of its dual partner, supersymmetric string waves, and by special properties of duality symmetry to preserve the condition of the embedding of the spin connection in a subgroup of a gauge group. Our generalized fundamental strings are different from the charged heterotic string solution obtained by Sen [8] by twisting the uncharged string solution

\footnotetext{
${ }^{15}$ As explained above, in the string case we have control only on anomaly-related $\alpha^{\prime}$ corrections.
} 
of [14]. For instance, in Sen's solution the charge-dependent terms in the metric occur in the $d u^{2}+d v^{2}$ sector, whereas in our case the $A_{i}$-dependent terms occur in the $d u d x^{i}$ sector. Another difference is that in our solution the antisymmetric tensor contains $A_{i}$-dependent terms, whereas in Sen's solution the antisymmetric tensor has no charge-dependent terms. Sen's solution is known to be supersymmetric in the zero slope limit [8, 25]. However, no information is available about the $\alpha^{\prime}$ corrections to this solution.

The advantage of our method of using sigma-model duality is in the fact that the structure of $\alpha^{\prime}$ corrections is under control for the dual solution if it was under control for the original

solution. In this way the nice properties of the plane waves are carried over to the string-type solutions via sigma model duality, acting as a symmetry of the target space action.

\section{Acknowledgements}

We are grateful to G. Horowitz for a useful discussion. The work of E.B.and R.K. was partially supported by a NATO Collaborative Research Grant. The work of E.B. has been made possible by a fellowship of the Royal Netherlands Academy of Arts and Sciences (KNAW). E.B. would like to thank the Physics Department of Stanford University for its hospitality. The work of R.K. was supported in part by NSF grant PHY-8612280. 


\section{A Notation and conventions}

We use a metric with mostly minus signature. Our conventions for the Riemann tensor and the spin connection are given in an appendix of [12]. We often use a light-cone basis for the ten-dimensional coordinates $x^{\mu}$ :

$$
x^{\mu}=\left(u, v, x^{i}\right), i=1, \ldots, 8, \quad u=\frac{1}{\sqrt{2}}(t+x), v=\frac{1}{\sqrt{2}}(t-x),
$$

where $t \equiv x^{0}$ and $x \equiv x^{9}$. In this paper, all the indices are raised and lowered with the full ten-dimensional metric $g_{\mu \nu}$. In the case in which the metric corresponds to the SSW solution given in eq. (21), the relation between upper and lower indices is

$$
\begin{aligned}
& \xi^{u}=\xi_{v}, \\
& \xi^{v}=\xi_{u}-\left(2 A_{u}+\sum_{i=1}^{8} A_{i}^{2}\right) \xi_{v}+\sum_{i=1}^{8} A_{i} \xi_{i}, \\
& \xi^{i}=A_{i} \xi_{v}-\xi_{i} .
\end{aligned}
$$

The constant Killing vectors $k^{\mu}$ and $l^{\mu}$ are given in the light-cone basis by:

$$
\begin{aligned}
k^{\mu} & =\frac{1}{\sqrt{2}}(1,-1,0, \ldots, 0), \\
l^{\mu} & =(0,1, \ldots, 0) .
\end{aligned}
$$

The expressions for these Killing vectors with down indices depend on the metric we are using. For the SSW metric (21) they are given by:

$$
\begin{aligned}
k_{\mu} & =\frac{1}{2} \sqrt{2}\left(2 A_{u}-1,1, A_{i}\right), \\
l_{\mu} & =(1,0, \ldots, 0) .
\end{aligned}
$$

The inner products between $k$ and $l$ for the SSW metric are given by

$$
l^{2}=0, \quad k^{2}=A_{u}-1, \quad k \cdot l=\frac{1}{2} \sqrt{2} .
$$

\section{B Proof of Supersymmetry of the Generalized FS Solu- tion}

In this appendix we will show that the generalized FS solution is supersymmetric. Since the supersymmetry transformation rules involve fermions it is necessary to reformulate the SSW Ansatz for the metric in terms of zehnbein fields:

$$
e_{\mu}^{a}=\delta_{\mu}^{a}+A_{\mu} l^{a}
$$


The unbroken supersymmetries of the SSW solution are given by

$$
l^{\mu} \gamma_{\mu} \epsilon_{0}=0 \quad \text { or } \quad\left(\gamma^{0}+\gamma^{9}\right) \epsilon_{0}=0,
$$

where $\epsilon_{0}$ is a constant ten-dimensional spinor. It is instructive to see how the sigma model duality transformation leads to unbroken supersymmetries for the generalized FS solution as well.

In order to investigate the existence of unbroken supersymmetries for our purely bosonic solutions we only need to consider the bosonic terms in the supersymmetry transformation rules of the fermions. They are given by

$$
\begin{aligned}
\delta \psi_{\mu} & =\left(\partial_{\mu}-\frac{1}{4} \Omega_{\mu+}^{a b} \gamma_{a b}\right) \epsilon \\
\delta \lambda & =\left(\gamma^{\mu} \partial_{\mu} \phi+\frac{1}{4} H_{\mu \nu \rho} \gamma^{\mu \nu \rho}\right) \epsilon \\
\delta \chi & =-\frac{1}{4} F_{\mu \nu} \gamma^{\mu \nu} \epsilon .
\end{aligned}
$$

We first consider the $\lambda$ transformation rule. Requiring that $\delta \lambda=0$ leads to the equation

$$
\gamma^{i}\left(\partial_{i} \phi\right) \epsilon+\frac{3}{2} H_{i u v} \gamma^{i u v} \epsilon+\frac{3}{4} H_{i j u} \gamma^{i j u} \epsilon=0,
$$

where it is understood that in this equation the generalized FS solution is substituted. To investigate this equation we need the form of the (inverse) zehnbein fields of the generalized FS solution. They are given by:

$$
\begin{aligned}
e_{a}^{u} & =\delta_{a}^{v}, \\
e_{a}^{v} & =\left(1-A_{u}\right) \delta_{a}^{u}-A_{i} \delta_{a}^{i} . \\
e_{a}^{i} & =\delta_{a}^{i} .
\end{aligned}
$$

It is now not too difficult to show that the first two and the last terms in (68) vanish separately provided that the supersymmetry parameter $\epsilon$ satisfies ${ }^{\text {to }}$

$$
\left(\gamma^{0}-\gamma^{9}\right) \epsilon=0 .
$$

We next consider the gravitino transformation rule. Instead of substituting the generalized FS solution into the equation $\delta \psi_{\mu}=0$ it is easier, and equivalent, to use the SSW solution and to require that after a duality transformation $\delta \psi_{\mu}=0$. This leads to the equation

$$
\delta \psi_{\mu}=\left(\partial_{\mu}-\frac{1}{4} \tilde{\Omega}_{\mu+}^{a b} \gamma_{a b}\right) \epsilon=0 .
$$

Applying the duality rotation of the torsionful spin connection given in (15) we find

$$
\partial_{\mu} \epsilon-\frac{1}{4} \Omega_{\mu+}{ }^{a b} \gamma_{a b} \epsilon+\frac{1}{k^{2}} k^{\lambda} k^{a} \Omega_{\lambda-, \mu}{ }^{b} \gamma_{a b} \epsilon=0,
$$

\footnotetext{
${ }^{16}$ Note that both (64) and (70) can be written as $\gamma^{u} \epsilon=0$, using curved indices.
} 
where it is now understood that the SSW solution is substituted. We next substitute the expression for the torsionful spin connections corresponding to the SSW solution [12]:

$$
\Omega_{\mu+}{ }^{a b}=-2 l^{[a} \mathcal{A}^{b]}{ }_{\mu}, \quad \Omega_{\mu-}{ }^{a b}=\mathcal{A}^{a b} l_{\mu},
$$

where $\mathcal{A}_{\mu \nu}=\partial_{\mu} A_{\nu}-\partial_{\nu} A_{\mu}$. We thus find that the following equation must be satisfied:

$$
\partial_{\mu} \epsilon+\frac{1}{2} l_{\rho} \mathcal{A}_{\sigma \mu} \gamma^{\rho \sigma} \epsilon+\frac{k \cdot l}{k^{2}} k_{\rho} \mathcal{A}_{\mu \sigma} \gamma^{\rho \sigma} \epsilon=0
$$

The $\mu=v$ component of this equation is automatically satisfied. Using the condition (70) we find that the $\mu=u$ component is satisfied as well. Finally, for $\mu=i$ we find that the equation is satisfied provided that

$$
\epsilon=\left(A_{u}-1\right)^{-1 / 2} \epsilon_{0}
$$

where $\epsilon_{0}$ is constant.

Finally, by using the condition (70) in the form $\gamma^{u} \epsilon=0$ and the fact that the gauge field corresponding to the generalized FS solution only has a nonvanishing $u$-component, it is not too difficult to show that also $\delta \chi=0$.

We thus conclude that the generalized FS solution has 8 unbroken supersymmetries given by eqs. (70) and (75). We should stress that it is not obvious that duality transformations always

preserve the supersymmetry of a given solution. Note that the unbroken supersymmetries before and after the duality transformation differ from each other.

\section{Proof of the space-time supersymmetry of the K-T So- lution}

The solution of [9] contains a flat metric describing a four-dimensional spacetime with coordinates $x^{\mu}=\left\{u, v, x^{1}, x^{2}\right\}$, a 2 -form field

$$
B=-2 f(u) d x^{1} \wedge d x^{2}
$$

and a $u$-dependent dilaton $\phi(u)$. The only nonzero component of $H$ is given by $H_{u 12}=-1 / 3 \partial_{u} f$.

The supersymmetry transformation rules for the dilatino

$$
\delta \lambda=\left(\gamma^{\mu} \partial_{\mu} \phi+\frac{1}{4} H_{\mu \nu \rho} \gamma^{\mu \nu \rho}\right) \epsilon=0
$$

have a non-trivial solution under the condition that

$$
\gamma^{u} \epsilon=0
$$


The supersymmetry transformation rules for the gravitino have a $v$ - component

$$
\delta \psi_{v}=\left(\partial_{v}-\frac{1}{4} \Omega_{v+}^{a b} \gamma_{a b}\right) \epsilon=0
$$

This equation is satisfied under the condition that $\epsilon$ is $v$-independent, since $\Omega_{v+}{ }^{a b}=0$. The $i$ component of this equation is solved if $\epsilon$ is $x^{i}$-independent and $\gamma^{u} \epsilon=0$ :

$$
\delta \psi_{i}=\left(\partial_{i}-\frac{1}{4} \Omega_{i+}{ }^{a b} \gamma_{a b}\right) \epsilon=0
$$

The $u$-component of this equation

$$
\delta \psi_{u}=\left(\partial_{u}+\frac{3}{4} H_{u}{ }^{12} \gamma_{12}\right) \epsilon=0
$$

is satisfied if the spinor of unbroken supersymmetry $\epsilon$ has a specific dependence on $u$-coordinate of the form

$$
\epsilon(u)=e^{\frac{1}{4} f(u) \gamma^{1} \gamma^{2}} \epsilon_{0}
$$

After a duality transformation in the $x^{1}$-direction, the dual solution is given by a zero axion field and the following non-flat metric:

$$
d s^{2}=2 d u d v+2 f(u) d x^{1} d x^{2}-d x^{1} d x^{1}-\left(1+f^{2}\right) d x^{2} d x^{2} .
$$

The dilaton field remains unchanged: $\tilde{\phi}=\phi$. The supersymmetry of the dual solution is proven as follows. The equation $\delta \lambda=0$ again leads to the condition that $\gamma^{u} \epsilon=0$. To investigate the supersymmetry transformation rule of the gravitino it is convenient to first consider the dual of $\Omega_{+}$:

$$
\tilde{\Omega}_{c+}{ }^{a b}=-\frac{3}{2}\left(H_{c}^{a b}-4 k^{[a} H_{1 c}^{b]}\right) .
$$

Here $k^{\mu}$ is the vector $k^{\mu}=(0,0,1,0)$. Using this expression it follows that the equation $\delta \psi_{v}=0$ is satisfied under the condition that $\epsilon$ is $v$-independent. Furthermore, using $\gamma^{u} \epsilon=0$, it follows that $\delta \psi_{i}=0$ if $\epsilon$ is $x^{i}$-independent. Finally, the equation $\delta \psi_{u}=0$ is satisfied if

$$
\delta \psi_{u}=\left(\partial_{u}-\frac{3}{4} H_{u}{ }^{12} \gamma_{12}\right) \epsilon=0
$$

or

$$
\epsilon(u)=e^{-\frac{1}{4} f(u) \gamma^{1} \gamma^{2}} \epsilon_{0}
$$

Finally, one can show that both before and after the duality transformation all the $T$-tensors and Lorentz Chern-Simons terms vanish. We therefore conclude that both the Klimčík-Tseytlin solution as well as its dual are supersymmetric to all orders in $\alpha^{\prime}$. 


\section{References}

[1] C.G. Callan, Jr., J.A. Harvey and A. Strominger, Supersymmetric String Solitons, proceedings of the 1991 Trieste Spring School on String Theory and Quantum Gravity (World Scientific, 1992).

[2] G.T. Horowitz, The Dark Side of String Theory: Black Holes and Black Strings, to appear in the proceedings of the 1992 Trieste Spring School on String Theory and Quantum Gravity.

[3] A. Sen, Black Holes and Solitons in String Theory, to appear in the proceedings of the 1992 ICTP Summer Workshop, Trieste, July 2-3.

[4] E. Bergshoeff and M. de Roo, Nucl. Phys. B328, 439 (1989).

[5] A. Strominger, Nucl. Phys. B343, 167 (1990); C.G. Callan, Jr., J.A. Harvey and A. Strominger, Nucl. Phys. B359, 611 (1991).

[6] T. Buscher, Phys. Lett. 159B, 127 (1985); ibid 194B, 59 (1987); ibid 201B, 466 (1988).

[7] G. Veneziano, Phys. Lett. B265, 287 (1991); K. Meissner and G. Veneziano, Phys. Lett. B267, 33 (1991); M. Gasperini and G. Veneziano, Phys. Lett. B277, 256 (1992).

[8] A. Sen, Phys. Lett. B271, 295 (1991); ibid. Phys. Lett. B274, 34 (1992); ibid. Nucl. Phys. B388, 457 (1992); S. Hassan and A. Sen, Nucl. Phys. B375, 103 (1992).

[9] C. Klimčík and A.A. Tseytlin, Duality invariant class of exact string backgrounds, preprint CERN-TH.7069/93.

[10] E. Kiritsis, Nucl. Phys. B405 (1993) 109; A. Kiritsis and A. Giveon, preprint hepth/9303016, Nucl. Phys. B in press; ibid. preprint hepth/9309064.

[11] D. Gershon, preprint hepth/9311122.

[12] E. Bergshoeff, R. Kallosh and T. Ortín, Phys. Rev. D47, 5444 (1993).

[13] J.H. Horne, G.T. Horowitz and A.R. Steif, Phys. Rev. Lett. 68, 568 (1992).

[14] A. Dabholkar, G. Gibbons, J. Harvey and F. Ruiz, Nucl. Phys. B340, 33 (1990).

[15] R. Güven, Phys. Lett. 191B, 265 (1987).

[16] D. Amati and C. Klimčík, Phys. Lett 219B, 443 (1988); D. Amati, M. Ciafaloni and Veneziano, Nucl. Phys. B347, 550 (1990).

[17] G.T. Horowitz and A. R. Steif, Phys. Rev. Lett. 64, 260 (1990); ibid. Phys. Rev. D42, 1950 (1990). 
[18] C. M. Hull and E. Witten, Phys. Lett. 160B, 398 (1985).

[19] M. Roček and E. Verlinde, Nucl. Phys. B373, 630 (1992), and references therein.

[20] A.H. Chamseddine, Nucl. Phys. B185, 403 (1981); E. Bergshoeff, M. de Roo, B. de Wit and P. van Nieuwenhuizen, Nucl. Phys. B195, 97 (1982); G.F. Chapline and N.S. Manton, Phys. Lett. 120B, 105 (1983).

[21] R.E. Rudd, Nucl. Phys. B352, 489 (1991).

[22] G.T. Horowitz, in Proceedings of Strings '90, College Station (World Scientific, 1991).

[23] H.W. Brinkmann, Proc. Natl. Acad. Sci. U.S. 9, 1 (1923).

[24] M. de Roo, M. Suelmann and A. Wiedemann, Nucl. Phys. 405, 326 (1993); M. Suelmann, Effective Actions for Heterotic String Theory, to appear in the proceedings of the Journées Relativistes '93.

[25] D. Waldram, Phys. Rev. D47, 2528 (1993). 